\title{
Concept Paper \\ Global Dynamic Response of a Medium-Sized Floating Offshore Wind Turbine with Stall Regulation
}

\author{
Moe Moe Aye ${ }^{1, *}$ and Uwe Ritschel ${ }^{1,2}$ \\ 1 Chair of Wind Energy Technology, University of Rostock, 18059 Rostock, Germany; \\ uwe.ritschel@uni-rostock.de \\ 2 IWEN Institut für Windtechnik, Energiespeicherung und Netzintegration gGmbH, 18119 Rostock, Germany \\ * Correspondence: moe.aye2@uni-rostock.de
}

check for updates

Citation: Aye, M.M.; Ritschel, U. Global Dynamic Response of a Medium-Sized Floating Offshore Wind Turbine with Stall Regulation. Energies 2022, 15, 166. https:// doi.org/10.3390/en15010166

Academic Editor: Davide Astolfi

Received: 10 November 2021

Accepted: 24 December 2021

Published: 27 December 2021

Publisher's Note: MDPI stays neutral with regard to jurisdictional claims in published maps and institutional affiliations.

Copyright: (C) 2021 by the authors. Licensee MDPI, Basel, Switzerland. This article is an open access article distributed under the terms and conditions of the Creative Commons Attribution (CC BY) license (https:// creativecommons.org/licenses/by/ $4.0 /)$.

\begin{abstract}
In this paper, a two-bladed medium-sized floating wind turbine with variable speed and power regulation by stall is studied. For floating offshore wind turbines, the major challenges are related to the dynamical behavior of the system in response to combined wind and wave loading. Especially for smaller systems, the coupling of aerodynamic and wave forces may lead to large amplitude motions. Coupled aero-hydro-servo-elastic simulations are carried out in OpenFAST. The goal of the study is to investigate the global dynamic response of the hypothetical wind turbine with stall regulation. Stall regulation concept is proposed and the structural loads are computed and results are presented and discussed.
\end{abstract}

Keywords: offshore wind turbine; stall regulation; aeroelastic simulation; variable speed operation; torque control; structural dynamic behavior

\section{Introduction}

Global climate change has the potential for more devastating impacts on lives on our planet. More frequent natural disasters have reminded us that we need to decarbonize our economics more urgently than ever to restore the balance. In most low-income countries especially Southeast Asian countries, clean and sustainable energy at an affordable price is highly demanded. Furthermore, land scarcity and population growth are giving a strong impetus for developers to venture into deep waters teeming with steadier wind resources, yet additional design challenges.

Motivated by the need for affordable energy supply, the concept of a medium-sized stall regulated wind turbine was developed within the framework of the research project CART in cooperation with the Chair of Wind Energy Technology the University of Rostock, several German companies, the Vietnam National University, and several companies in Vietnam [1]. Floating substructures are usually considered in the context of large wind turbines. What, then, is the reason to consider a medium-sized wind turbine? Medium-sized wind turbines have a great potential in terms of low cost, low impact on the environment, and being able to be deployed for isolated communities like remote villages or islands for decentralized supply of electrical energy. While the cost of energy may not be lower than the ones for large offshore wind turbines, it might be lower than the cost of other technologies like diesel generators or other types of generation.

Certainly, several concepts of stall regulated both horizontal-axis wind turbines and vertical-axis wind turbines have been developed and their benefits over pitch regulation have been highlighted. However, horizontal-axis FOWT with variable-speed stall regulation have yet to be developed and the concept needs to be verified thoroughly. For floating offshore wind turbines like CART, the major challenges are related to the dynamical behavior of the system in response to combined wind and wave loading and the choice of power regulation. The wind turbine needs to have an efficient power regulation that can optimize power output, provide the highest possible efficiency in normal operation, and ensure safe operation during 
grid loss or storm conditions. Of equal importance, power regulation must ensure that the structural loads are reduced especially when exposed to constantly changing impact from the environment like wind gusts and turbulence. Moreover, the coupling of aerodynamic forces, structural forces, and hydrodynamic forces can lead to large amplitude motions. In the present paper, the proposed stall regulation concept is briefly explained and the global dynamic response of the investigated wind turbine is studied.

\section{The CART System}

\subsection{Stall Regulation}

The CART machine utilizes a direct-drive generator system, in which the rotor is directly coupled with the generator. The generator type is a gearless PMSG with a rated generator speed of $43 \mathrm{rpm}$ and a rated mechanical power of $144 \mathrm{~kW}$, in which the rotor speed can be varied throughout the operating regions. For stall-regulated wind turbines, the blade twist angle plays a major role, especially in the stall regime. Therefore, the stall progression along the blade was adjusted so that the rotational speed is kept nearly constant after stall.

To formulate an appropriate control strategy, it is a prerequisite to understand how the rotor torque changes with variation in rotor speed at different wind speeds as depicted in Figure 1. It is evident from the torque-speed curve that the rotor speed increases continuously with the increase in wind speed. Therefore, a control concept is necessary to ensure that the turbine operates safely under all operating modes. The black line in Figure 1 depicts the proposed ideal torque-speed curve of CART. The black dotted curve represents the maximum power coefficient curve of the CART wind turbine. In the partial load region, the torque controller attempts to keep tip speed ratio at optimum level by changing the rotor speed using the torque-speed curve defined by Leithead and Connor (2000) [2] as in the following Equation (1). The torque curve follows the trajectory of the maximum power curve in the partial load region until the maximum speed for this region is reached, which is $95 \%$ of rated rotor speed in our case, as schematically shown in Figure 1 . To alleviate high-frequency excitations of the control system [3], the measured generator speed is filtered before it is fed back. For this, a first-order recursive low pass filter is implemented, which is based on the filter used in the NREL 5 MW wind turbine baseline controller. The cutoff frequency of the filter is set to approximately one-fourth of the blade's first edgewise natural frequency.

$$
T_{a}=k \Omega_{o}^{2}
$$

where, the torque curve coefficient $k$ can be determined according to Equation (2)

$$
k=\frac{1}{2 \lambda_{o}^{3}} \rho_{a} \pi R^{5} C_{p, \max }
$$

where, $\lambda_{o}$ stands for optimum tip speed ratio, $\rho_{a}$ for air density, $R$ for the rotor radius, and $C_{p, \max }$ for the maximum power coefficient of the rotor.

In the full load region, another different torque control algorithm was proposed to limit the rotor speed not to exceed its maximum allowable speed in the full load region. Similar to the partial load region, the generator torque was implemented as a function of rotor speed. The measured speed is fed back as input directly into the torque controller. To alleviate high-frequency excitations of the control systems, the same first-order recursive low pass filter is implemented similar to the partial load region. Whenever the generator speed exceeds the predefined generator speed setpoint in the full load region, the turbine requests a higher generator torque than the current value. The current generator torque is estimated by tracking the $\mathrm{Cp}$,max curve continuously and adding part of the torque found at maximum $\mathrm{Cp}$,max to it. The amount of additional torque is obtained by multiplying part of the torque found at maximum $\mathrm{Cp}$, max by a variable slope. The value of the slope and the predefined generator speed setpoint can be varied depending on the size of the generator and the converter rating. Furthermore, the generator torque is limited not to 
exceed $35 \%$ above the value of the rated torque in order to prevent requesting high torque, which can otherwise cause overheating and ultimately lead to system failure. In this way, the rotor speed is limited so as not to exceed the allowed maximum rotational speed of the rotor in above-rated regions. The implemented generator control algorithm is briefly described in Table 1.

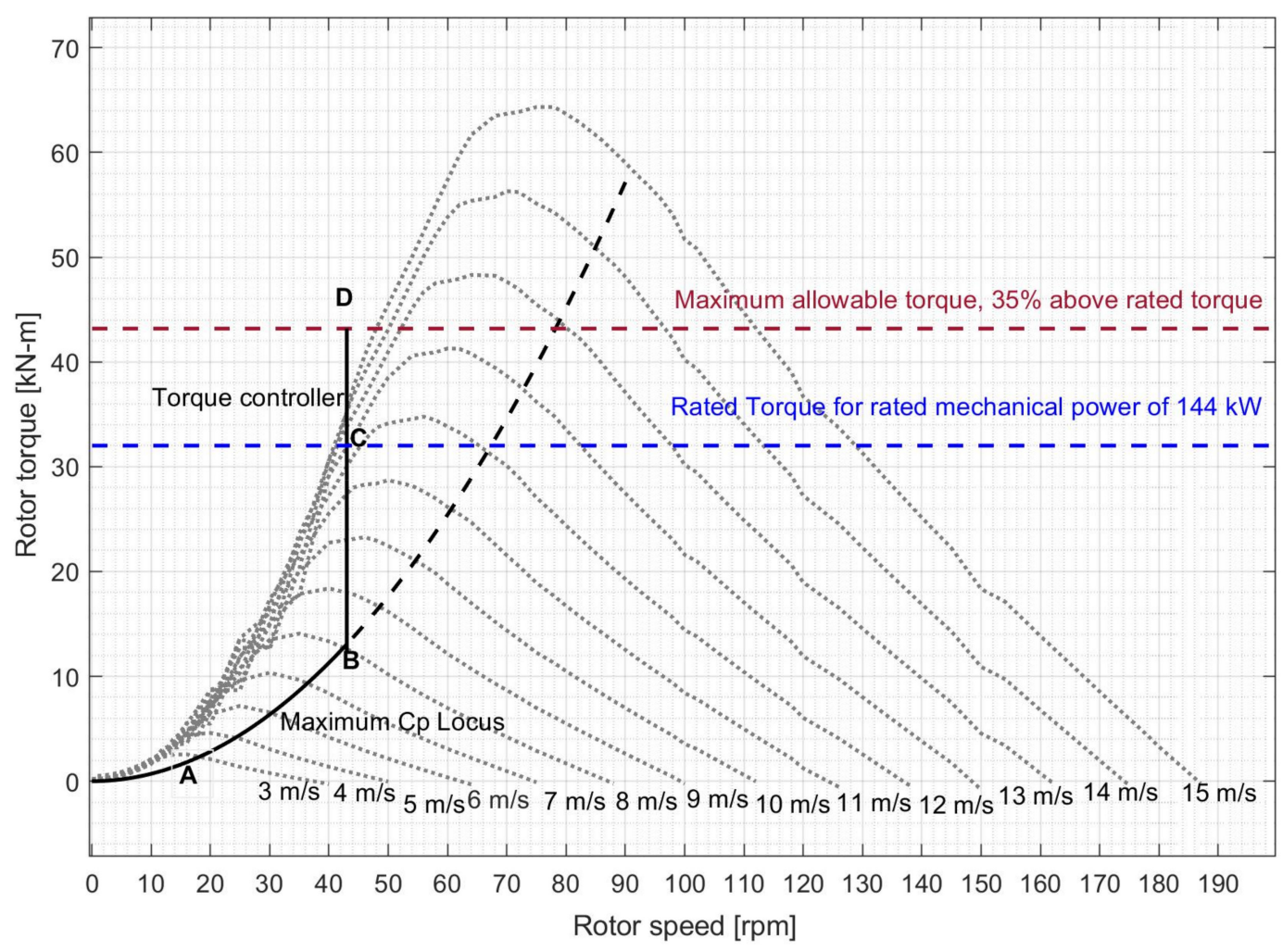

Figure 1. Rotor torque as function of rotor speed at variable wind speed.

Table 1. Implemented control algorithm.

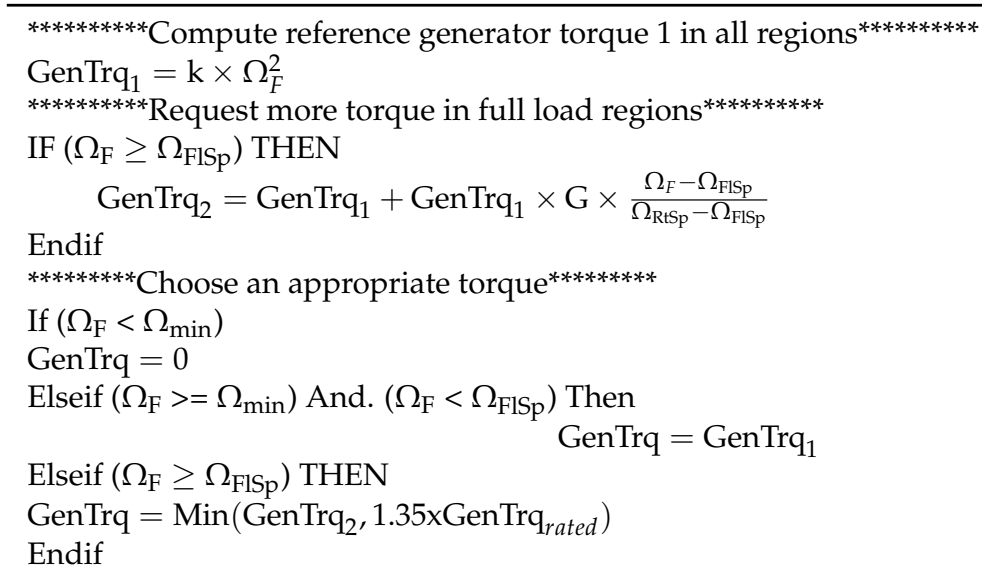

where,

$\Omega_{\mathrm{F}}=$ filtered generator speed

$\Omega_{\min }=$ minimum generator speed at which the torque controller should start working

$\Omega_{\mathrm{FlSp}}=$ rotational speed of the generator in full load region

$\Omega_{\mathrm{RtSp}}=$ rated rotational speed of the generator

GenTrq ${ }_{1}=$ generator torque in partial load regions

GenTrq $_{2}=$ generator torque in full load regions 
GenTrq $\mathrm{q}_{\text {rated }}=$ rated generator torque

GenTrq = selected generator torque

$\mathrm{k}=$ torque curve coefficient

$\mathrm{G}=$ variable slope

\subsection{Semisubmersible Floater}

The CART wind turbine was mounted on a semisubmersible floater, which was developed by [1,4]. The floater consists of three columns: the largest one is the tower column, where the tower is attached, and the other two columns of the same size are offset columns (see Figure 2). Each column is located at a radius of $18 \mathrm{~m}$ from the center of the floater and arranged with an angular distance of $120^{\circ}$ from each other. The columns are connected to each other with main horizontal braces. The vertical diagonal bracing between main horizontal braces provides vertical stiffness and horizontal diagonal bracing between main horizontal braces provides horizontal stiffness to resist the lateral loads from wind and wave. The tower column is $10 \mathrm{~m}$ tall and the height of offset columns is $9 \mathrm{~m}$. The floater has a draft of $7.6 \mathrm{~m}$ and this left $2.4 \mathrm{~m}$ of freeboard. Heave plates were installed at the bottom of each column in order to reduce heave, pitch, and roll motions. Figure $2 b$ depicts the main dimensions of the floater.

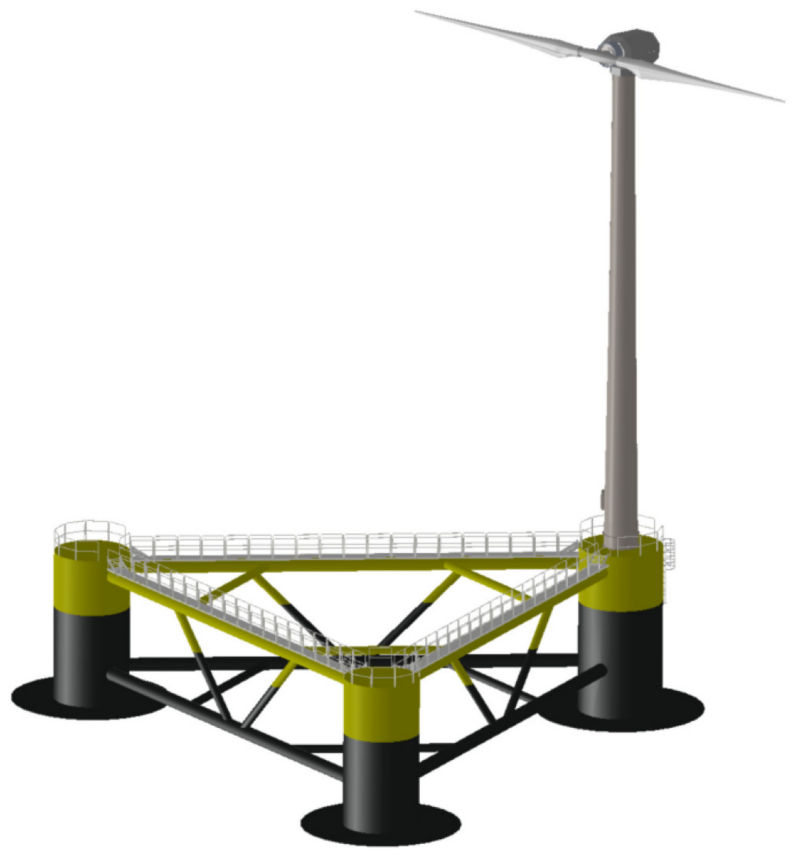

(a)

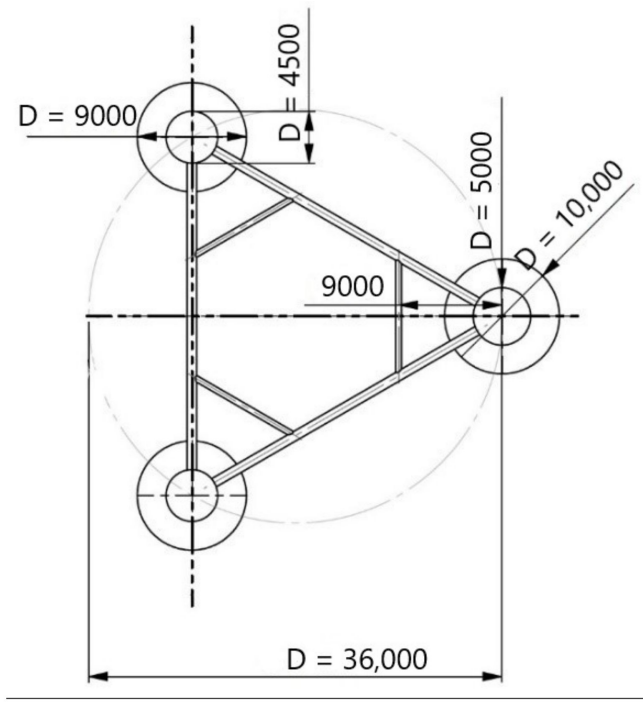

(b)

Figure 2. An impression CART wind turbine mounted on semisubmersible platform (a) and main dimensions of the floater $(\mathbf{b})[4]$.

\subsection{Design Load Cases}

Coupled aero-hydro-servo-elastic simulation was carried out in OpenFAST v2.2.0 [5] developed by the National Renewable Energy Lab (NREL). Following the work of [6,7], design load cases (DLC) as specified in the design requirements for offshore wind turbines by IEC [8] were divided into normal operating conditions, extreme conditions with faults, and severe conditions with the worst consequences in the present study. Table 2 lists the summary of selected design loads cases along with their wind models, wave models, partial safety factor (PSF), and number of load cases (NLC). 
Table 2. Selected design load cases.

\begin{tabular}{|c|c|c|c|c|c|}
\hline DLC & Wind Condition & Wave Conditions & Events & PSF & NLC \\
\hline \multicolumn{6}{|c|}{ (1) Normal operating condition } \\
\hline 1.1 & NTM, $V_{\text {in }}<V_{\text {hub }}<V_{\text {out }}$ & $\mathrm{NSS}, \mathrm{H}_{\mathrm{s}}=\mathrm{E}\left[\mathrm{H}_{\mathrm{S}} \mid \mathrm{V}_{\mathrm{hub}}\right], \beta=0^{\circ}$ & Normal & 1.25 & 180 \\
\hline 1.2 & NTM, $V_{\text {in }}<V_{\text {hub }}<V_{\text {out }}$ & NSS, $\mathrm{H}_{\mathrm{s}}=\mathrm{E}\left[\mathrm{H}_{\mathrm{s}} \mid \mathrm{V}_{\mathrm{hub}}\right], \beta=0^{\circ}$ & Normal & 1.0 & 180 \\
\hline 1.3 & ETM, $V_{\text {in }}<V_{\text {hub }}<V_{\text {out }}$ & NSS, $H_{\mathrm{s}}=\mathrm{E}\left[\mathrm{H}_{\mathrm{s}} \mid \mathrm{V}_{\mathrm{hub}}\right], \beta=0^{\circ}$ & Normal & 1.35 & 180 \\
\hline 1.4 & $\mathrm{ECD}, \mathrm{V}_{\mathrm{hub}}=\mathrm{V}_{\mathrm{r}}-2 \mathrm{~m} / \mathrm{s}, \mathrm{V}_{\mathrm{r}}, \mathrm{V}_{\mathrm{r}}+2 \mathrm{~m} / \mathrm{s}$ & $\mathrm{NSS}, \mathrm{H}_{\mathrm{s}}=\mathrm{E}\left[\mathrm{H}_{\mathrm{s}} \mid \mathrm{V}_{\mathrm{hub}}\right], \beta=0^{\circ}$ & Normal & 1.35 & 486 \\
\hline \multicolumn{6}{|c|}{ (2) Extreme conditions with fault } \\
\hline 1.6 & NTM, $\mathrm{V}_{\text {in }}<\mathrm{V}_{\text {hub }}<\mathrm{V}_{\text {out }}$ & SSS, $\mathrm{H}_{\mathrm{s}}=\mathrm{H}_{\mathrm{s} 50}, \beta=0^{\circ}$ & Normal & 1.35 & 180 \\
\hline 2.3 & EOG, $V_{\text {hub }}=V_{r} \pm 2 \mathrm{~m} / \mathrm{s}, V_{\text {out }}$ & $\mathrm{NSS}, \mathrm{H}_{\mathrm{s}}=\mathrm{E}\left[\mathrm{H}_{\mathrm{s}} \mid \mathrm{V}_{\mathrm{hub}}\right], \beta=0^{\circ}$ & Grid loss & 1.10 & 12 \\
\hline 6.1 & EWM, $V_{\text {hub }}=V_{\text {ref }}$ & ESS, $\mathrm{H}_{\mathrm{s}}=\mathrm{H}_{\mathrm{s}, 50}, \beta=0^{\circ}, \pm 30^{\circ}$ & Yaw $=0, \pm 8^{\circ}$ & 1.35 & 54 \\
\hline \multicolumn{6}{|c|}{ (3) Extreme conditions with the worst consequences } \\
\hline 2.1 & NTM, $V_{\text {in }}<V_{\text {hub }}<V_{\text {out }}$ & $\mathrm{NSS}, \mathrm{H}_{\mathrm{s}}=\mathrm{E}\left[\mathrm{H}_{\mathrm{s}} \mid \mathrm{V}_{\mathrm{hub}}\right], \beta=0^{\circ}$ & Grid loss & 1.35 & 60 \\
\hline 2.2 & NTM, $V_{\text {in }}<V_{\text {hub }}<V_{\text {out }}$ & $\mathrm{NSS}, \mathrm{H}_{\mathrm{s}}=\mathrm{E}\left[\mathrm{H}_{\mathrm{s}} \mid \mathrm{V}_{\mathrm{hub}}\right], \beta=0^{\circ}$ & Faults in yaw system & 1.10 & 370 \\
\hline $6.2 \mathrm{a}$ & EWM, $V_{\text {hub }}=V_{\text {ref }}$ & ESS, $H_{s}=H_{s, 50}, \beta=0^{\circ}, \pm 30^{\circ}$ & $180^{\circ}<$ Yaw $<180^{\circ}$ & 1.10 & 108 \\
\hline 6.3 & EWM, $V_{\text {hub }}=V_{\text {ref }}$ & $\mathrm{ESS}, \mathrm{H}_{\mathrm{s}}=\mathrm{H}_{\mathrm{s}, 1}, \beta=0^{\circ}, \pm 30^{\circ}$ & $-20^{\circ}<$ Yaw $<20^{\circ}$ & 1.35 & 54 \\
\hline $7.1 \mathrm{a}$ & EWM, $V_{\text {hub }}=V_{\text {ref }}$ & ESS, $H_{s}=H_{s, 1}, \beta=0^{\circ}, \pm 30^{\circ}$ & Yaw $=0, \pm 8^{\circ}$ & 1.10 & 108 \\
\hline 7.2 & NTM, $V_{\text {in }}<V_{\text {hub }}<V_{\text {out }}$ & $\begin{array}{c}\text { NSS, } H_{\mathrm{s}}=\mathrm{E}\left[\mathrm{H}_{\mathrm{S}} \mid \mathrm{V}_{\mathrm{hub}}\right] \\
\beta=0^{\circ}, \pm 30^{\circ}\end{array}$ & Yaw $=0, \pm 8^{\circ}$ & 1.35 & 380 \\
\hline
\end{tabular}

\section{Simulation Results}

\subsection{Response of Controller and Platform Motions as a Function of Wind Speed}

It is important to understand the performance of the system and motion responses under steady-state conditions before conducting a series of load cases. The goal is to examine the mean offset of floater motions and the response of controller, which helps to enhance the controller design to keep the platform motion within a safe range. During steady wind simulations, a constant uniform wind inflow is applied perpendicular to the rotor plane for $400 \mathrm{~s}$ and no wave and current loads are considered. The turbine was operating with a generator torque controller to optimize the power in the partial load region and limit the power in the full load region.

Figure 3 shows the mean values of the response of the controller and platform motion as a function of wind speeds. In partial load regions, the turbine operates with optimal power coefficient $\mathrm{Cp}$ to extract the maximum power from the wind, whereas the mean value of $\mathrm{Cp}$ decreases for higher wind speeds as expected (see Figure 3). Similarly, the generator torque increases quadratically with the mean wind speed (see Figure 3a) and the generator power increases cubically with the mean wind speed in partial load regions (see Figure $3 \mathrm{c}$ ). In the full load region, the generator torque increases until the rated generator power is reached and decreases after that when it enters into the stall region.

Since the turbine uses stall regulation, the mean rotor thrust force increases with the increase in wind speeds (see Figure 3d). Special attention needs to be paid in extreme conditions since there is no active aerodynamic control to reduce the thrust force and hence this can affect the dynamic response of the substructure. In Figure 3c, the mean mechanical power of the onshore and offshore CART system are compared. It was found that the mechanical power of the offshore CART system is slightly lower than the land-based one. This is due to the pitch motion of the floater, which consequently causes the rotor plane deviate from the inflow wind direction. Figure $3 e, f$ shows how the mean offset of the platform motion changes with the increase in wind speeds. Comparing Figure $3 \mathrm{e}, \mathrm{f}$ with Figure $3 d$, it was found that the surge and pitch motions increase with the increase in aerodynamic thrust force on the rotor. Especially in high winds, the large platform motion 
causes the rotor plane to deviate from the incoming wind. As a result, the rotational speed is decreased and there is a change in power output. This can also imply that the CART system is more likely to be affected by cut-out load cases since the rotor blades are forced to stall at high winds.

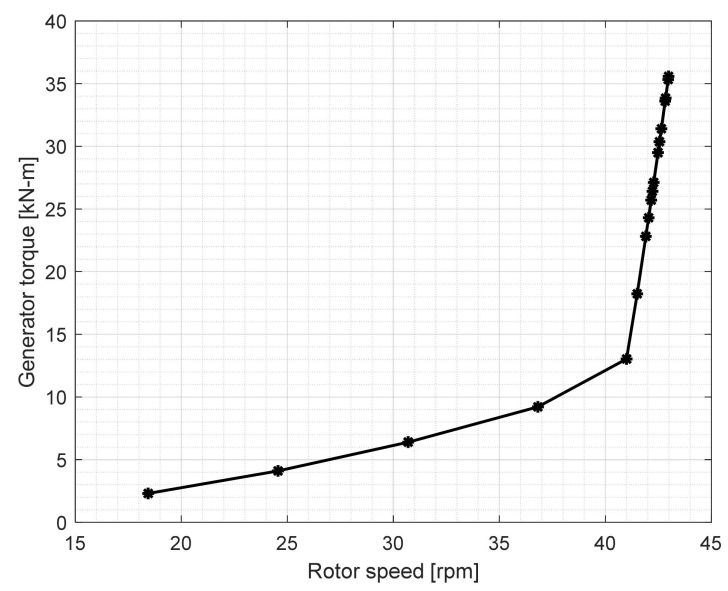

(a)

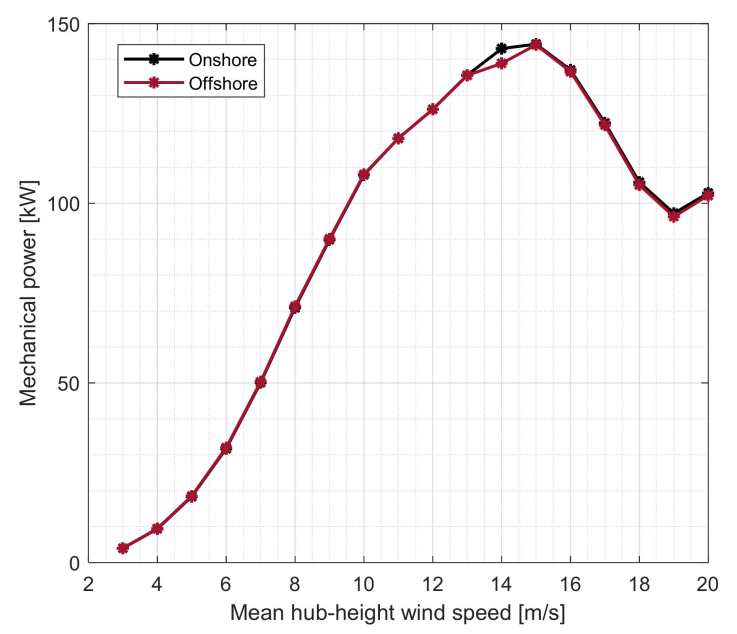

(c)

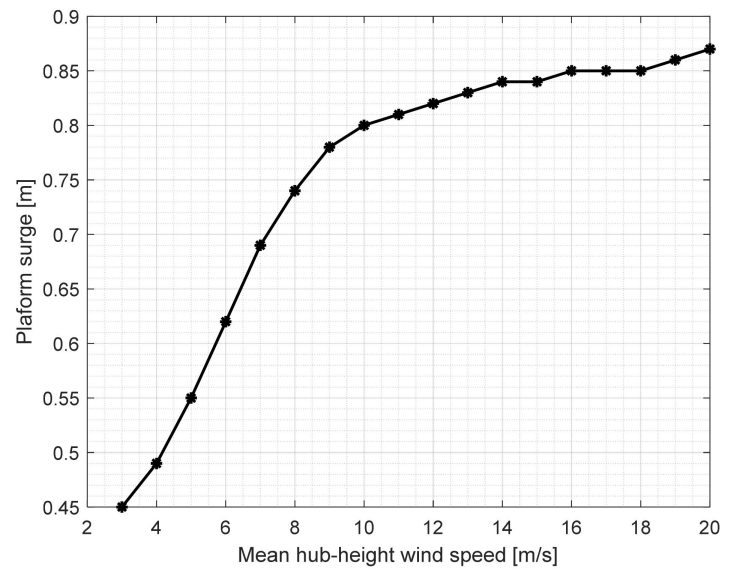

(e)

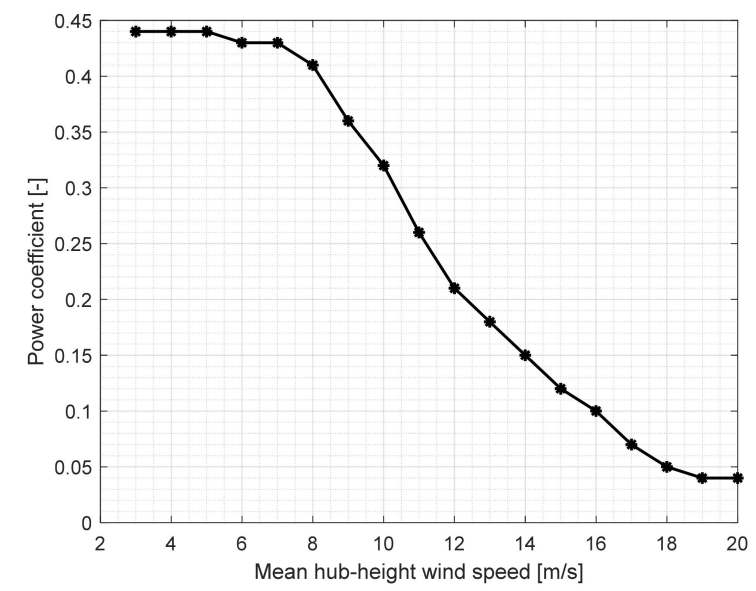

(b)

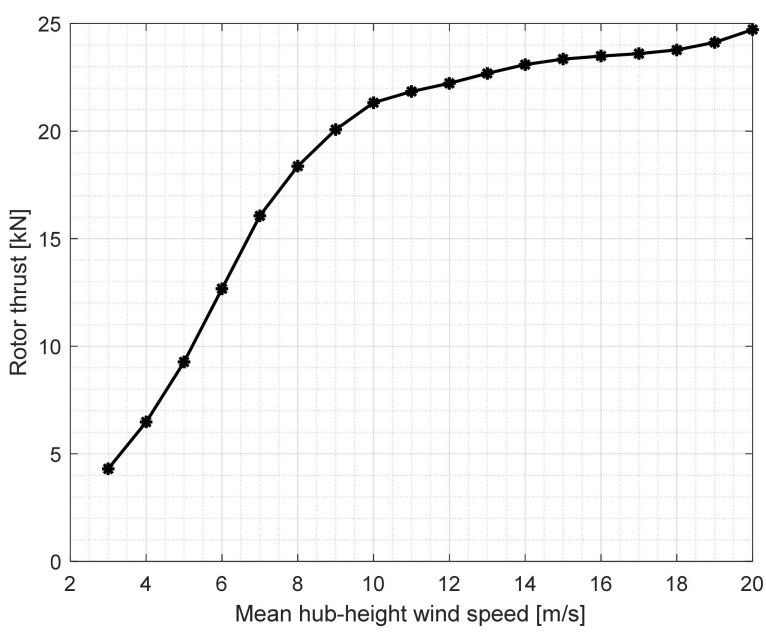

(d)

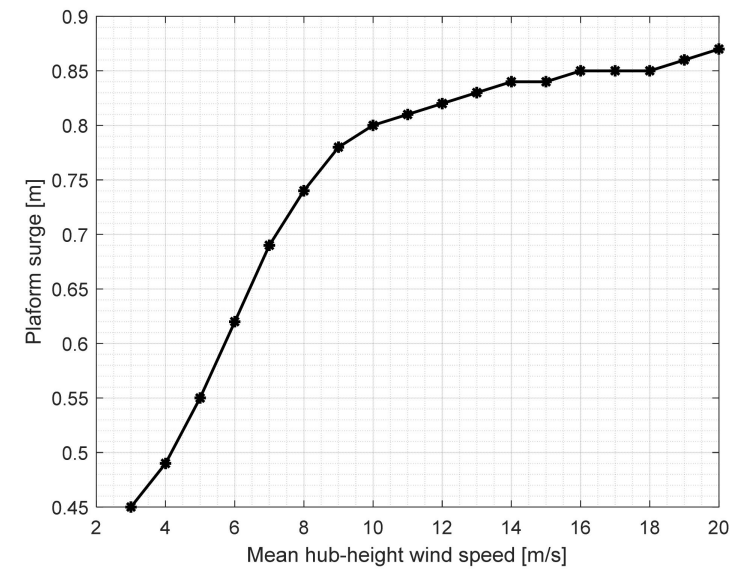

(f)

Figure 3. Torque-speed curve (a), response of power coefficient (b), generator power (c), rotor thrust (d), platform surge (e), and platform pitch (f). 


\subsection{Response in Normal Operating Conditions}

By examining the extreme loads generated by all DLCs during normal operations, it was interesting to note that the out-of-plane blade deflection and blade root moments were driven by DLC 1.4, which simulates extreme wind direction change (ECD), in both the onshore and offshore CART systems. The discussion made in the present section will mainly focus on the offshore CART system. Figures 4 and 5 compare the time history of a particular event which triggers the most extreme loads in blade root bending moment at blade 2 denoted by RootMyc2 and out-of-plane blade deflection denoted by TipDxc1 for the offshore CART system. In the figures, the red line represents simulation number 6 and the blue one represents simulation number 431. In the figures, the $x$-axis represents time and the $y$-axis represents the quantity of each load parameter. These results were generated directly from OpenFAST output file. Therefore, they are not weighted by PSFs.

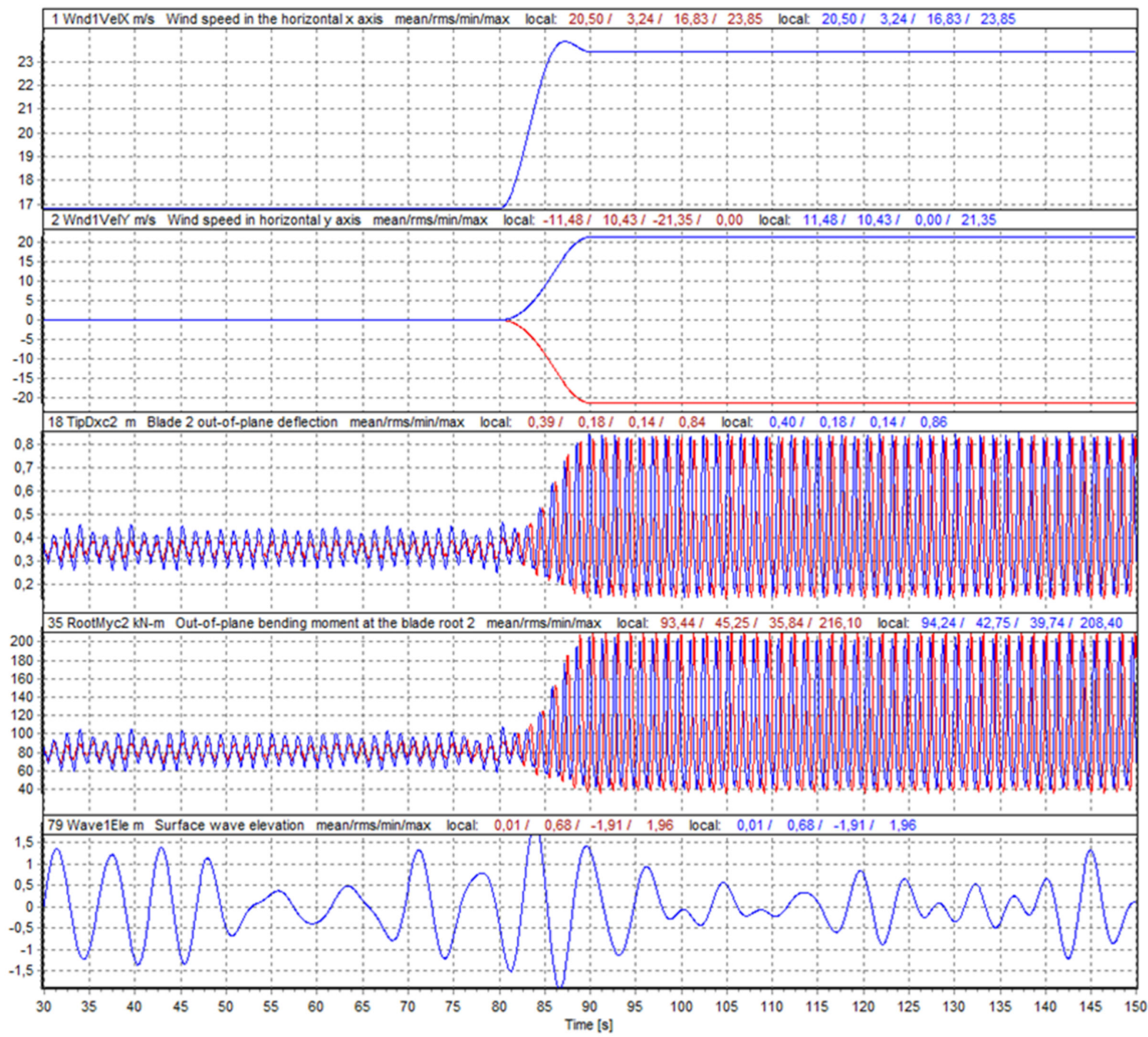

Figure 4. Time histories from DLC 1.4 simulation number 6 (shown in red) and simulation number 431 (shown in blue) for the offshore CART system (a).

Simulation number 431 was run with an IEC discrete wind model ECD-r+2 considering no yaw misalignment, slope of vertical inflow of $8^{\circ}$, and an initial azimuth angle of $90^{\circ}$, whereas simulation number 6 with ECD $+r+2$, a yaw misalignment of $8^{\circ}$, slope of vertical inflow of $-8^{\circ}$, and an initial azimuth angle of $0^{\circ}$. The change in wind direction is $42.35^{\circ}$ to the right for simulation number 431 and the same magnitude to the left for simulation 
number 6 . The maximum out-of-plane deflection was found at blade two in simulation number 431 at a time of $142.59 \mathrm{~s}$, wind speed of $23.42 \mathrm{~m} / \mathrm{s}$, and azimuth angle of $202^{\circ}$. The maximum blade root deflection occurs at blade two in simulation number 6 at a time $144.27 \mathrm{~s}$, wind speed of $23.42 \mathrm{~m} / \mathrm{s}$ and azimuth angle of $1.73^{\circ}$. In this case, blade two is pointing almost vertically down into the wind and the blade experiences the tensile force. By observing both cases, it seems that the blade deflection and blade root bending moment are sensitive to the wind speed close to the turbine cut-out wind speed. This is because the rotor thrust increases with the increase in wind speed. Furthermore, it is obvious from the figure that the floater pitch motion is influenced by wave elevation as it moves with the surface wave which is common for semisubmersible platforms. Rotor speed, generator torque, and generator power go up with the rise in wind speed as the ECD event starts. Eventually, transient behavior was observed in the rotor speed, generator torque, generator power, blade tip deflection, and blade root bending moment. Although it shows that the wind gust does not influence on pitch motion, it does influence on floater yaw motion. It is visible as a perturbing in floater yaw angle during wind gust, followed by a gentle yaw to the wind direction when the event is over. Negative yaw angle indicates the reverse movement of the platform. The difference in power production between these two load cases reflect that the power production is higher when the platform moves in the wind direction whereas the production is lower when the platform move from the wind direction (see Figure 4). It is obvious that DLC 1.4 is a particular event that is primarily influenced by wind gust and extreme change in wind direction. The influence of wave-induced loads on structural loads seems insignificant.

In order to gain more insights into the differences in dynamic response of the offshore and onshore CART system, Figure 6 compares the time history of wind speed, tower bottom side-to-side bending moment, tower bottom fore-aft moment, tower bottom torsional moment, tower top side-to-side displacement and nacelle acceleration between the offshore and onshore system. The red line represents the onshore CART system and the blue line represent the offshore CART system. The results were based on the simulation at mean hub-height wind speed of $20 \mathrm{~m} / \mathrm{s}$ from DLC 1.1 which corresponds to the cut-out wind speed of the turbine. Due to the difference in hub height, the time series of wind speed is not exactly identical between them. It is evident that the acceleration of the nacelle in the $y$-direction significantly decreased for the offshore CART system. However, the acceleration of the nacelle in the z-direction was found to be significantly larger for the offshore system. This might be related to the coupling between platform pitch and heave motion. As a result, tower bottom fore-aft and torsional moment increased for the offshore system. In order to get a better understanding of the correlation among wave loads, the tower vibration and the nacelle acceleration, Figure 7 compares Fast Fourier transform (FFT) diagrams of nacelle acceleration along the $y$-axis and z-axis between the onshore and offshore CART system. Regarding the nacelle acceleration along the y-axis for the onshore CART system, the peak energy was found to be mainly due to tower vibration for the onshore system as represented by the red line and $1 \mathrm{P}$ rotor frequency and $2 \mathrm{P}$ rotor blade passing frequency are also visible. In contrast, the nacelle acceleration along the y-axis seems be excited by wave loads, $2 \mathrm{P}$ and $4 \mathrm{P}$ rotor harmonic frequencies, and the tower vibration for the offshore system. However, the response amplitude of tower eigenfrequency was observed to be somewhat smaller than that of the onshore system, and the tower vibration and nacelle acceleration obviously decreased. As a result, tower bottom side-to-side moment decreased for the offshore CART system. Regarding the nacelle acceleration along the $\mathrm{z}$-axis, the largest amount of energy was found to be the wave energy range for offshore system, whereby the response amplitude is quite large. Apart from wave excitation forces, the nacelle acceleration was found to be dominated by the platform heave motion, rotor harmonic frequencies. This might be related to the coupling between platform pitch and heave motion. As a result, tower bottom fore-aft moment and tower bottom torsional moment increased for the offshore CART system. 


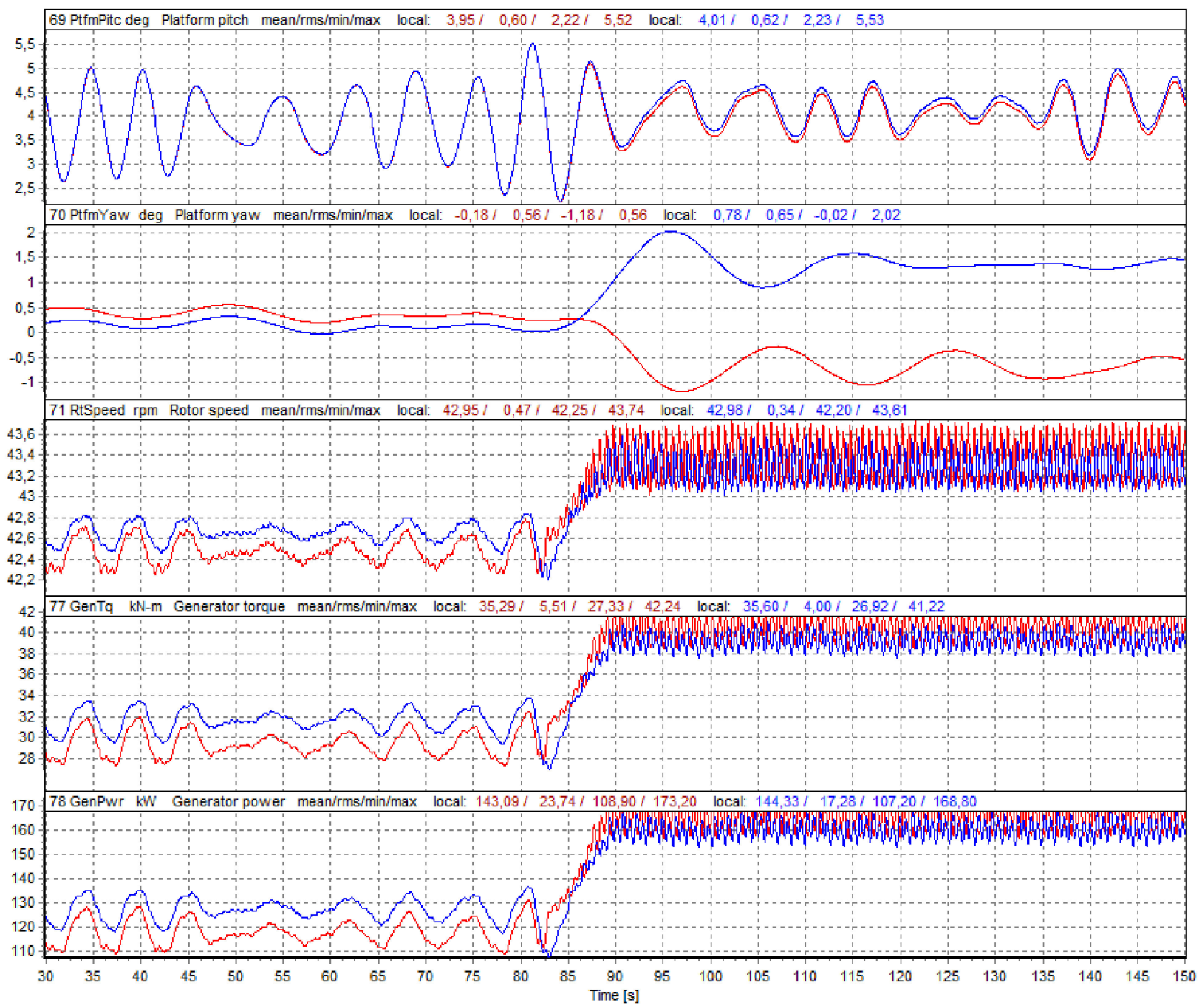

Figure 5. Time histories from DLC 1.4 simulation number 6 (shown in red) and simulation number 431 (shown in blue) for the offshore CART system (b).

\subsection{Response in Extreme Conditions with Faults}

By examining the response of both the onshore and offshore CART in extreme conditions, it was found that the extreme loads of several load components increased in the presence of floating substructure. For the offshore CART system, it was observed that DLC 2.3 contributes only a few parameters, namely, blade tip deflections, in-plane moment at the blade root, edgewise bending moment at the blade root, tower top yaw moment, and tower base axial force. On the other hand, DLC 6.1, which simulates storm condition and extreme sea states characterized by extreme wave heights, greatly influences the platform motions. It also contributes most parameters, namely, blade root bending moments, flapwise bending moment at the blade root, rotor thrust force, tower top side-to-side displacement, tower top bending moment, tower bottom fore-aft and side-to-side moments, and tower bottom torsional moment. It is quite common for a wind turbine with stall regulation that the highest rotor thrust force occurs in this load case. 


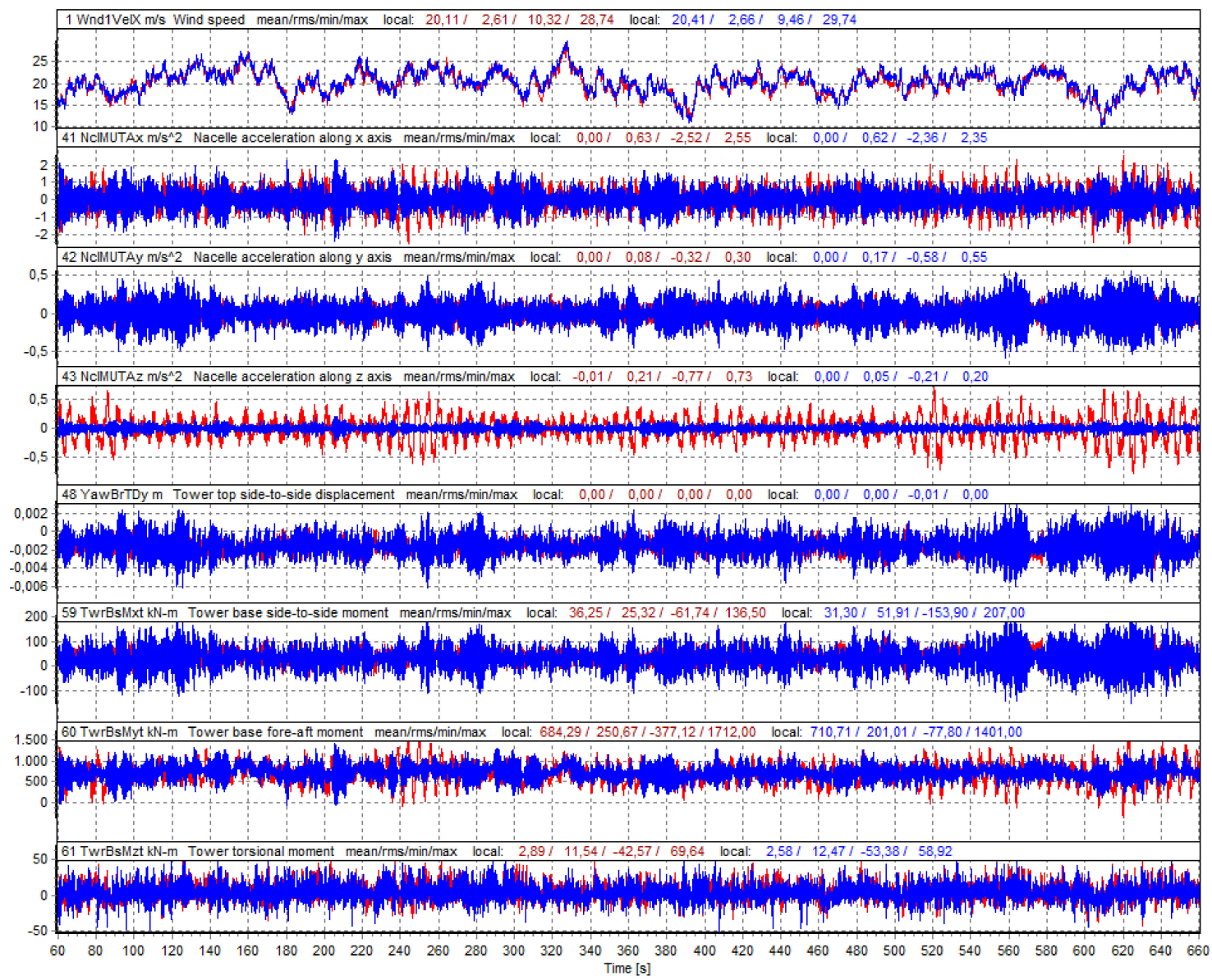

Figure 6. Comparison of time histories of wind speed, nacelle accelerations, tower top side-to-side displacement, tower base side-to-side moment, tower base fore-aft moment, and tower torsional moment between the onshore (shown in red) and offshore (shown in blue) wind turbine.

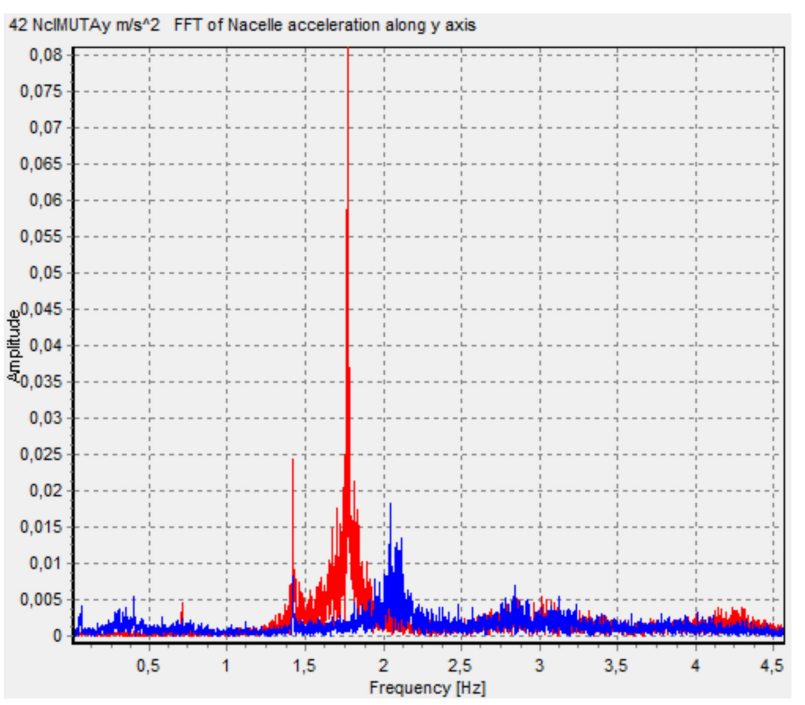

(a)

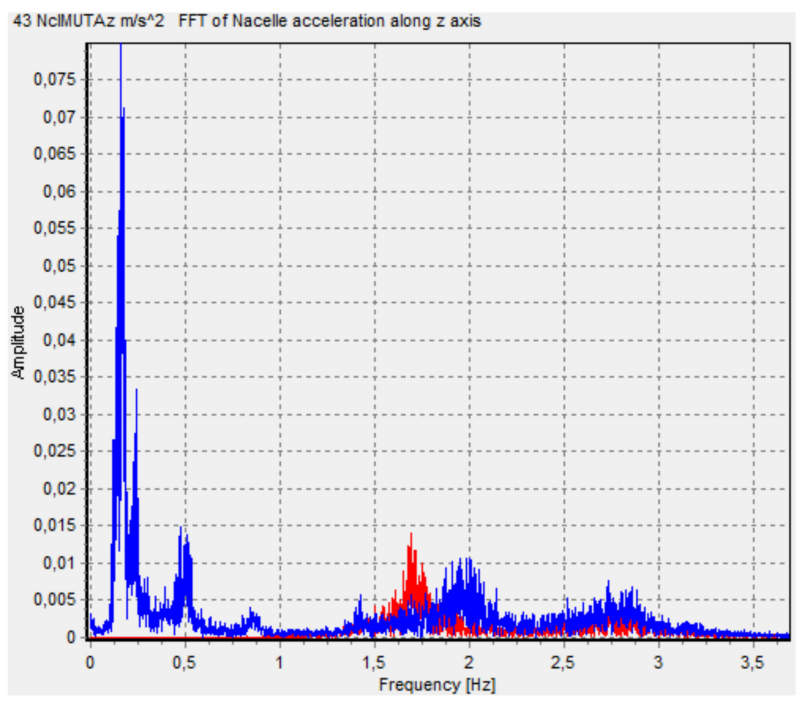

(b)

Figure 7. Comparison of FFT of nacelle acceleration along the $y$-axis (a) and $z$-axis (b) between the onshore (shown in red) and offshore (shown in blue) wind turbine. 


\subsubsection{Extreme Surge Motion in Extreme Wind and Wave Conditions}

The maximum surge motion was observed in simulation number 29 of DLC 6.1, which simulates the situation when the turbine is parked in storm conditions and an extreme sea state with a 50-year return period. The maximum surge motion of $3.19 \mathrm{~m}$ was driven by the particular simulation, in which no yaw misalignment was taken into account, but wind and wave misalignment of $30^{\circ}$ was considered. The maximum surge motion occurs at a wind speed $53.27 \mathrm{~m} / \mathrm{s}$ (see Figure 8). Mooring line 3 corresponds to the upwind mooring line and tension at fairlead of the mooring line is denoted by T3. The platform pitch and surge motion are coupled even in the absence of wind and wave forces. In this particular case, peak surge motion originates from the change in rotor thrust force, which is caused by the platform pitch motion. It is interesting to note that peak surge motion occurs when the rotor thrust abruptly decreases and the rotor thrust force increases again when the surge motion reaches its maximum value. What we can see more in the figure is that peak surge motion is also correlated to the tensions in the mooring lines which, in turn, is dominated by wind turbulence. When the platform is being pushed downward, the mooring line in the upwind side is being pulled upwards and large surge motion occurs. In order to get a better understanding of the correlation among wind, tension in mooring line upwind, and peak surge motion, the FFT of tension at fairlead of the mooring line 3 and surge motion are depicted in Figure 9.

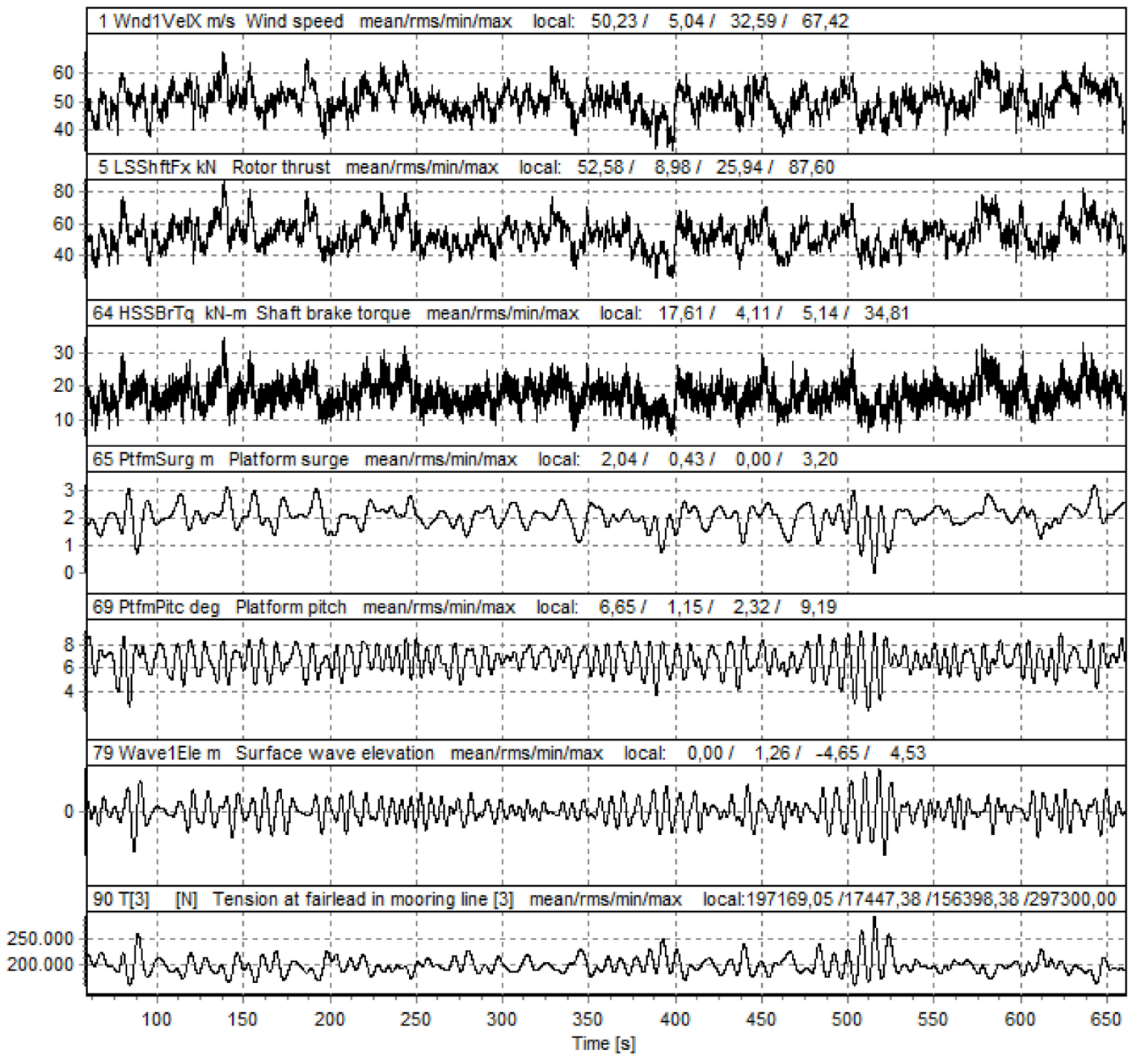

Figure 8. Time history of maximum surge motion, simulation number 3 of DLC 6.1. 


\subsubsection{Extreme Pitch Motion in Extreme Wind and Wave Conditions}

As mentioned above, the investigated semisubmersible platform is quite sensitive to large amplitude platform motions especially when it is subjected to large incident waves during severe sea states. When the turbine is parked, the mechanical shaft brake is engaged. Extreme platform pitch motion was observed in simulation number 18 of DLC 6.1. This particular case was simulated under consideration of a yaw misalignment of $8^{\circ}$ and the directionality of wind and wave being colinear. Extreme pitch motion occurs at a wind speed of $55.78 \mathrm{~m} / \mathrm{s}$ and time $531.37 \mathrm{~s}$. It appears that the sudden change in rotor thrust leads to large pitch motion as the pitch restoring stiffness is usually low for semisubmersible platforms. This results in a pitch angle of $9.10^{\circ}$ in storm conditions, which is just below the static inclination of $12^{\circ}$ for survival conditions prescribed by [9]. It was observed that the platform operates with a mean pitch angle of $6.62^{\circ}$ with a standard deviation of $1.04^{\circ}$ in this situation. Since the shaft brake is applied, the rotor is not in operational mode, and thus no generator torque and subsequently no power is produced. As we can clearly see in the time history of pitch motion in Figure 10, the platform keeps moving with the surface wave. Applying the shaft brake stops only the rotor from spinning. To reduce the platform motion in such conditions, it is desirable to integrate structural control systems like tuned mass damper.

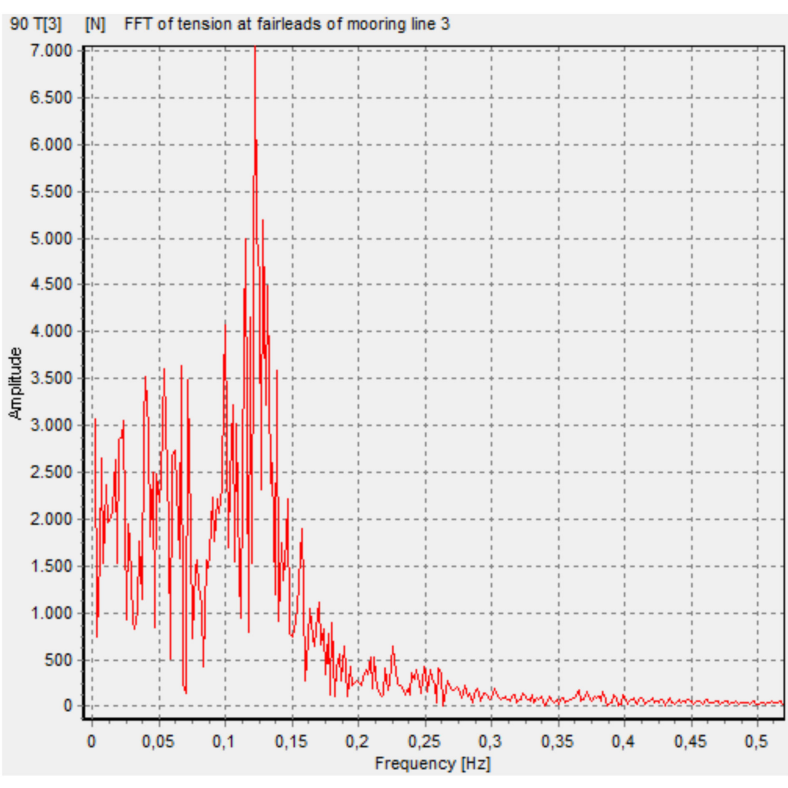

(a)

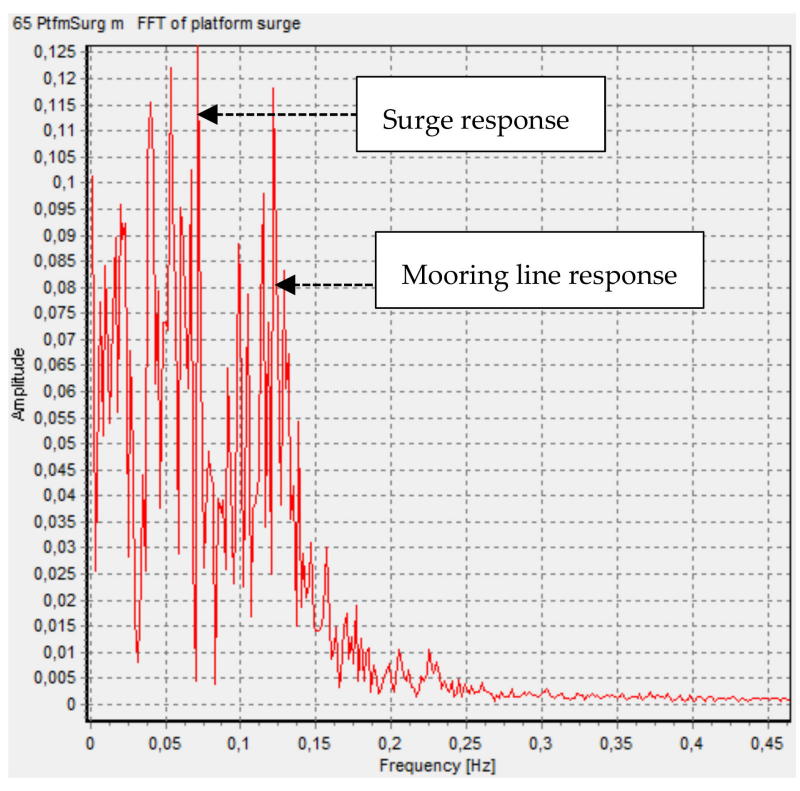

(b)

Figure 9. FFT of tension at fairlead in mooring line 3 (a) and surge motion (b).

To gain more insight into the dynamic response of the platform, FFT diagrams of wave and pitch motion are displayed in Figure 11, capturing the important peaks. As it is evident in the figure, the largest peak in the FFT diagram of pitch motion represents the structural natural frequency in pitch direction which is $0.12 \mathrm{~Hz}$. Low peak before the largest peak represents the response in the wind energy range. As it can be clearly seen in FFT of wave elevation, the wave peak frequency ranges between $0.12 \mathrm{~Hz}$ and $0.24 \mathrm{~Hz}$. It means that the wave frequency coincides with the structural frequency of the platform in pitch direction. To shift the natural frequency of pitch platform motion away from wave frequency, one way is to increase the structural stiffness in pitch direction. The other way is to incorporate a structural control system between the tower and the platform as mentioned above. 


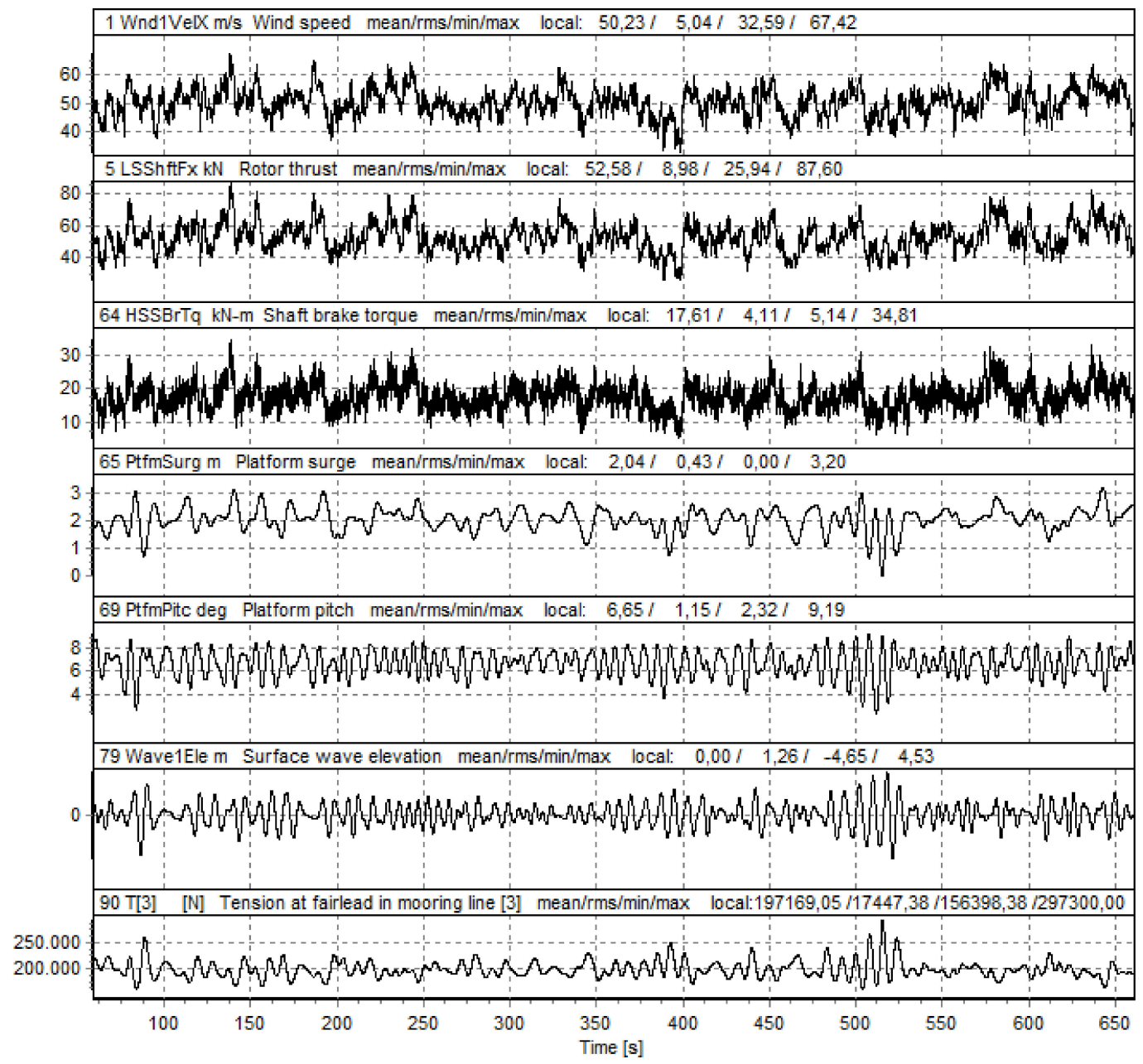

Figure 10. Time history of extreme pitch motion in simulation number 18 of DLC 6.1.

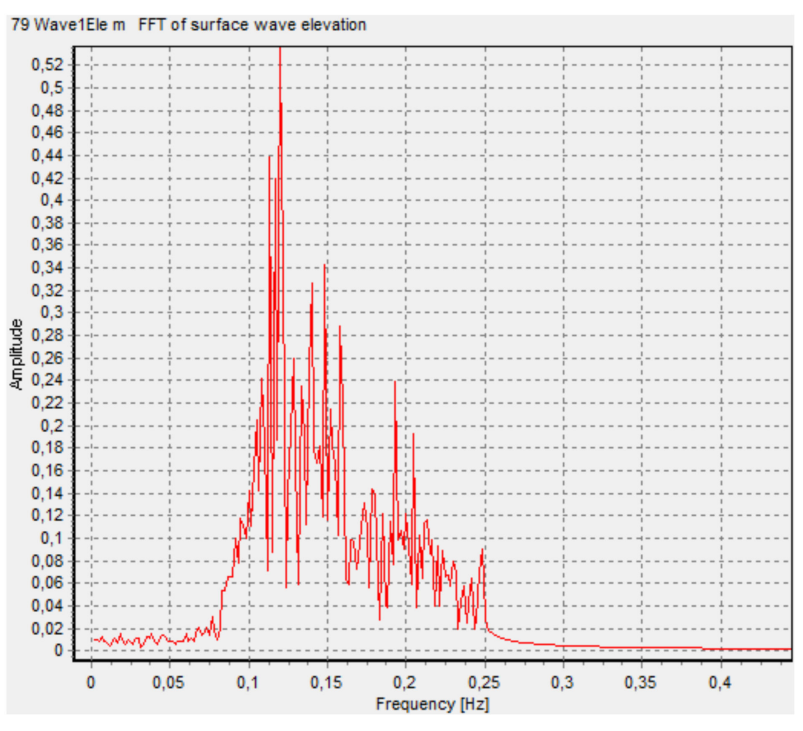

(a)

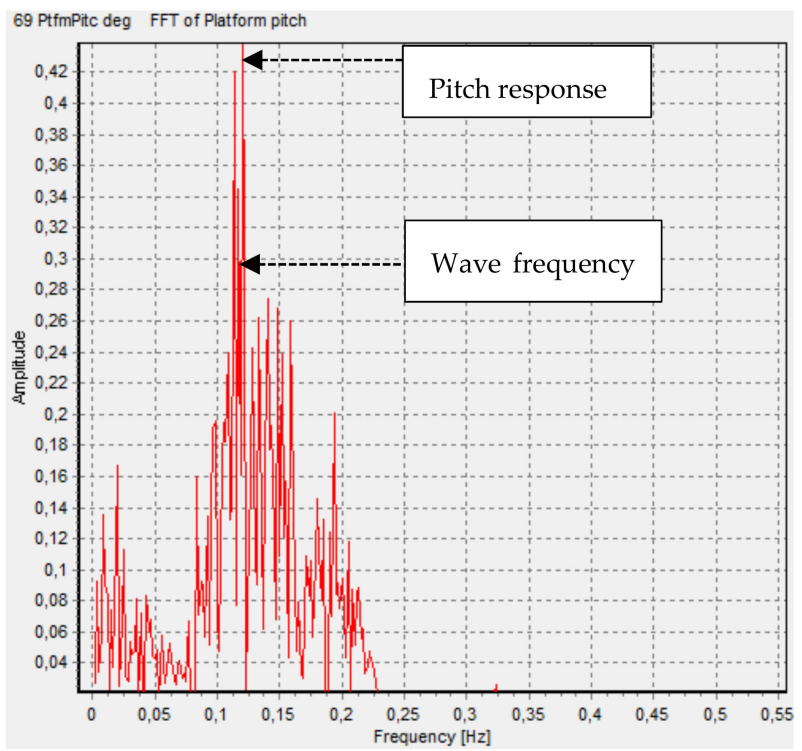

(b)

Figure 11. FFT of wave surface elevation (a) and pitch motion (b). 


\subsection{Response in Faults Events with Worst Consequences}

Based on the findings, the extreme tower bottom loads for the offshore CART system were driven by DLC 6.3, which simulates the turbine being parked in extreme wind and wave conditions when wind and wave are misaligned with an angle of $\pm 30^{\circ}$ and 1-year recurrence were assumed. On the other hand, the extreme tower bottom loads for the onshore CART system were driven by DLC 6.2 and DLC 2.2. The former simulates the turbine being parked in extreme wind condition with 50-year recurrence and the latter deals with faults in the yaw system during normal wind conditions.

\subsubsection{Influence of Wind-Wave Misalignment on Platform Motions}

To examine the influence of wind-wave misalignment on platform motions, Figure 12 compares time history of simulation number 73 shown in blue and simulation number 1 shown in red of DLC 6.2, where the latter was run without wind and wave misalignment but keeping other boundary conditions the same. Besides faults in the yaw system, it was observed that wind-wave misalignment leads to extreme platform yaw motion in the parked condition. The nacelle acceleration in the y-direction significantly increased when the wind and wave are misaligned in $-30^{\circ}$. It was observed that the platform sway, roll, and yaw motions significantly increased, whereas the platform surge and pitch motions slightly decreased. As a result of the change in wind-wave misalignment, tensions in the mooring lines had changed. Consequently, platform sway, roll, and yaw motions changed significantly. The mean platform yaw motion and standard deviation for the case with wind-wave misalignment are, respectively, about 1.12 times and 1.64 times larger than that for the case in which wind and wave misalignment is not considered. The mean platform sway motion and standard deviation for the case with wind-wave misalignment are, respectively, about 1.1 times and 2.07 times larger than that for the case in which wind and wave misalignment is not considered. Similarly, the mean platform roll motion and standard deviation for the case with wind-wave misalignment are, respectively, about 1.14 times and 7.08 times larger than that for the case in which wind and wave misalignment is not considered.

\subsubsection{Influence of Current Loads on Platform Motions}

To examine the influence of current loads on the platform motions, simulation number 1 of DLC 6.2 was rerun by disregarding the current loads. Figure 13 compares time history of the platform motions and nacelle accelerations between the load case, with current loads shown in blue and without current loads shown in red. As already mentioned, simulation number 1 was run under assumption of a yaw misalignment of $110^{\circ}$, no wind and wave misalignment, and $90^{\circ}$ of current direction.

Similar to wind-wave misalignment, it was observed that current loads and direction can change tension in the mooring lines. As a result, there is a significant increase in the platform sway, roll, and yaw motions, but a slight decrease in platform surge and pitch motions. The mean platform sway motion for the case with current loads is about 2.5 times larger than that for the case without current loads. Similarly, the mean platform roll motion for the case with current loads is about 1.83 times larger than that for the case without current loads whereas the standard deviation for the case with current loads is about 8.94 times larger than that for the case without current loads. The mean platform yaw motion and standard deviation for the case with current loads increased as much as about 1.4 times and 2 times larger, respectively, than that for the case without current loads. 


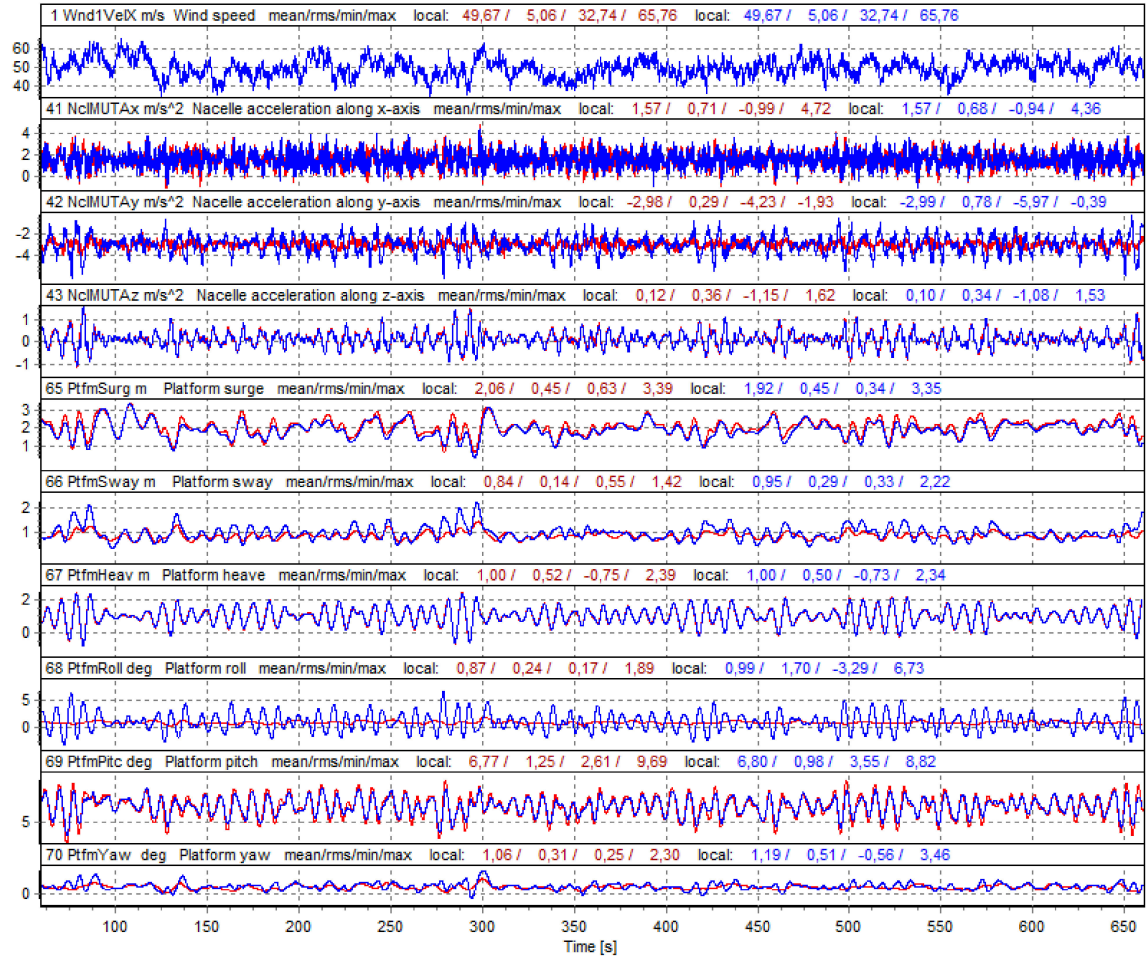

Figure 12. Time history of platform motions and nacelle acceleration from simulation with wind-wave misalignment (shown in red) and simulation without wind-wave misalignment (shown in blue).

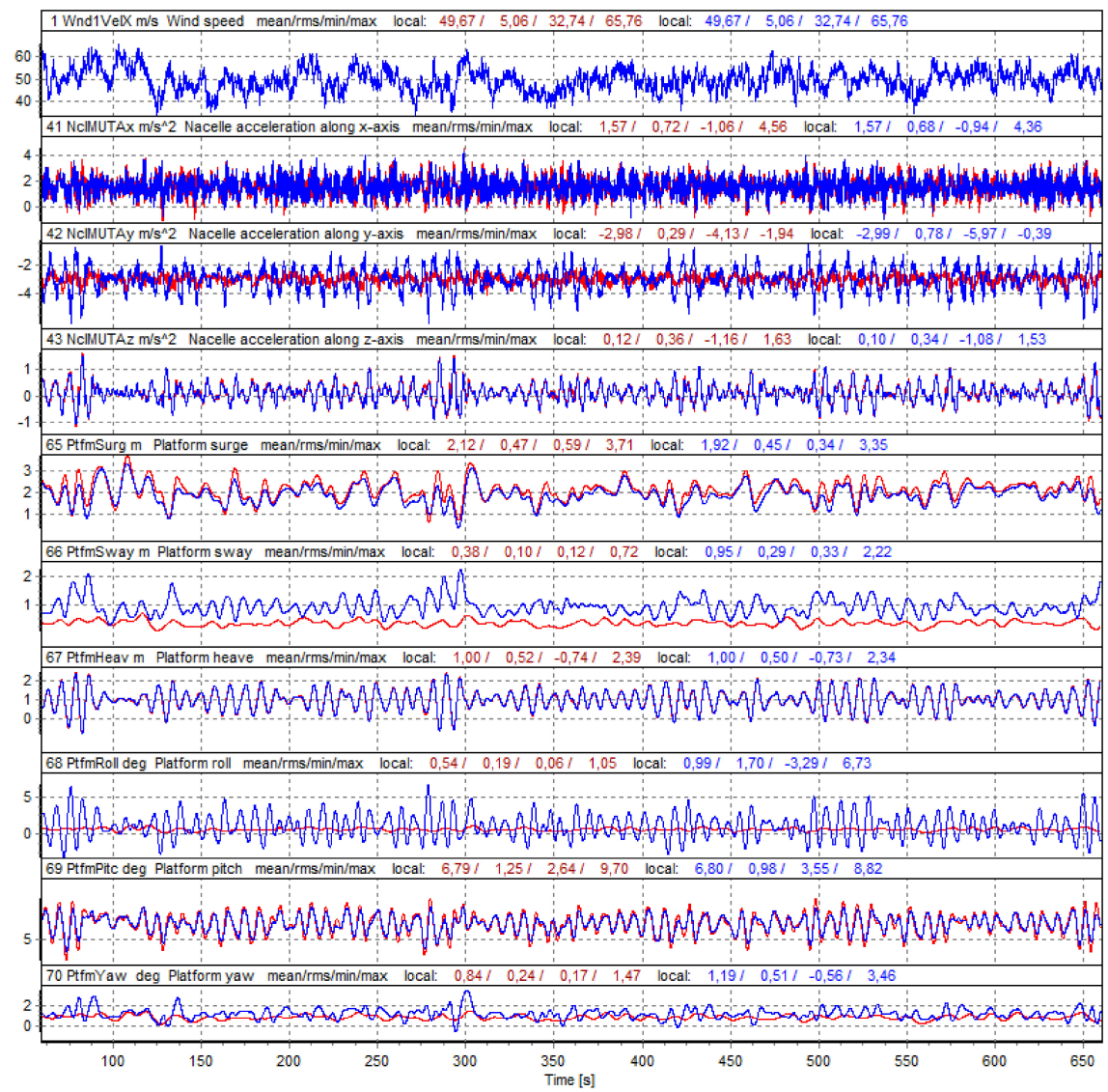

Figure 13. Time history of platform motions and nacelle acceleration from simulation without current loads (shown in red) and simulation with current loads (shown in blue). 


\section{Discussion}

In the present study, the global dynamic behavior of a medium-sized floating offshore wind turbine with stall regulation was investigated. The proposed stall regulation concept was briefly explained. The feasibility of the developed control concept was evaluated by simulating the turbine under steady wind conditions. Based on the resulted mean value, standard deviation, and maximum values of the response of the control system, the implemented torque control concept seems to be stable for the given control parameters. The result showed that the CART system is more likely to be affected by cut-out load cases since the rotor blades are forced to stall at high winds.

Because stall-regulated wind turbines have no active aerodynamic control system to reduce the rotor thrust force in high winds, it was observed that the load responses-more specifically, the mean values of most parameters-increase with the increase in wind speed in normal operations. The same applies to the load excursions of several parameters. On top of this, platform pitch motion contributes large fluctuations in power output and generator torque in high winds. Furthermore, large load excursions in tower top fore-aft displacement and tower bottom fore-aft moment of the offshore CART system might be related to an inverted pendulum effect which is mainly contributed from inertial forces due to tower deflection or platform motions. Regarding platform motions in normal operations, the extreme sway, heave, roll, pitch, and yaw motion appear at simulations in DLC 1.4, whereas the surge motion seems to be triggered by the extreme turbulence in DLC 1.3. Based on the findings, it was correlated to the fact that the tensions in the upwind mooring line are greatly influenced by wind turbulence.

By comparing the nacelle acceleration at cut-out wind speed, it is observed that the acceleration of the nacelle in the y-direction significantly decreased for the offshore CART system. However, the acceleration of the nacelle in the $\mathrm{z}$-direction was found to be significantly larger for the offshore system. This might be related to the coupling between platform pitch and heave motion. As a result, tower bottom fore-aft and torsional moment significantly increased for the offshore system.

Load analyses for extreme situations with faults showed that DLC 6.1 defining storm conditions and extreme sea states characterized by high wave heights greatly influence the platform motions. By examining on the load response in extreme condition with worst consequences, it was observed that extreme loads of most parameters were significantly increased in the presence of floating substructure. Upon comparing the resulted extreme loads among normal operation, fault events, and fault events with worst consequences, it was found that the extreme loads of most parameters were triggered by extreme events with faults. The comparison of different load cases confirmed that the heave, pitch, and roll motions are greatly affected by wave-induced motions, whereas surge, sway, and yaw motions are affected by motions in the mooring system, which in turn is dominated by wind turbulence. Moreover, wind, wave, and current misalignment leads to a significant increase in platform sway, roll, and yaw motions, whereas it leads to a slight decrease in platform surge and yaw motions. Similar to wind-wave misalignment, it was observed that current loads and direction can change tension in the mooring lines. As a result, there is a significant increase in the platform sway, roll, and yaw motions but a slight decrease in platform surge and pitch motions.

Author Contributions: Writing, M.M.A.; review and editing, U.R. All authors have read and agreed to the published version of the manuscript.

Funding: This research received no external funding.

Institutional Review Board Statement: Not Applicable.

Informed Consent Statement: Not Applicable.

Data Availability Statement: Not Applicable.

Conflicts of Interest: The authors declare no conflict of interest. 


\begin{abstract}
Abbreviation
CART: Compact and Robust Medium-sized Wind Turbine; ECD, Extreme coherent gust with direction change; EOG, Extreme operating gust; ETM, Extreme turbulence model; EWM, Extreme wind model; ESS, Extreme Sea state; FOWT, floating offshore wind turbine; NSS, Normal Sea State; NTM, Normal Turbulence Model; PMSG, permanent magnet synchronous generator.
\end{abstract}

\title{
References
}

1. Adam, F.; Schroeter, M.; Manh, V.V.; Dierken, P. CART-A Compact and Robust Wind Turbine Design for South Asia; Taylor \& Francis Group: Lisbon, Portugal, 2019.

2. Leithead, W.E.; Connor, B. Control of variable speed wind turbines: Design task. Int. J. Control 2000, 73, 1189-1212. [CrossRef]

3. Jonkman, J.; Butterfield, S.; Musial, W.; Scott, G. Definition of a 5-MW Reference Wind Turbine for Offshore System Development (No. NREL/TP-500-38060); National Renewable Energy Laboratory (NREL): Golden, CO, USA, 2009.

4. Schröter, M. Basis Design und KonstruktionsplanunEiner Schwimmenden Unterstruktur für eine Windenergieanlage Mittlerer Größe für den Südasiatischen Raum. Master's Thesis, University of Rostock, Rostock, Germany, 2018. (Unpublished).

5. OpenFAST. Available online: https://openfast.readthedocs.io/en/dev/source/user/aerodyn-olaf/Introduction.html (accessed on 1 January 2021).

6. G.L. Guidelines for the Certification of Wind Turbines; Germanischer Lloyd: Hamburg, Germany, 2012.

7. Jonkman, J.M. Dynamics Modeling and Loads Analysis of an Offshore Floating Wind Turbine (No. NREL/TP-500-41958); National Renewable Energy Laboratory (NREL): Golden, CO, USA, 2007.

8. IEC TS, 61400-3. Wind Energy Generation Systems-Part 3-2: Design Requirements for Floating Offshore Wind Turbines; International Electrotechnical Commission: Geneva, Switzerland, 2019.

9. DNVGL. DNV-GL-OS-C301. Stability and Watertight Integrity. Edition July. 2020. Available online: https://rules.dnv.com/docs/ pdf/DNV/OS/2017-01/DNVGL-OS-C301.pdf (accessed on 1 January 2021). 